\title{
Shoulder joint flexibility in top athletes
}

\author{
Hassan Daneshmandi", Farhad Rahmaninia, Hossein Shahrokhi, Pegah Rahmani, Saeid Esmaeili
}

Guilan University, Rasht, Iran.

Email: Danesh@guilan.ac.ir, Daneshmandi ph@yahoo.com

Received 17 April 2010; revised 17 May 2010; accepted 22 May 2010.

\begin{abstract}
Joint range of motion (ROM) is very important in daily activities, sport and in clinical diagnosis. Many factors have been reported to influence joint ROM. Musculoskeletal adaptation and some special side effects due to his or her physical demands and movement patterns in professional athletes are very important subjects in sport sciences. The present study is a comparison of shoulder joint ROM in elite athlete and non-athlete groups and its relationship to their age, post and years of their tournament play. The subjects in this study included 106 men with mean age $(23.65 \pm 3.45)$ and mean years of tournament play $(5.60 \pm 2.23)$. The groups of subjects included 26 handball players, 25 volleyball players, 25 soccer players and 30 non-athletes. Data were collected through questionnaires and inspection, and subjects' ROM was measured by "Leighton flexometer" $(r=0.90-0.99)$ in external rotation and abduction of shoulder joint in dominant and non-dominant hands. Data were analyzed by correlation coefficient, $\mathrm{t}$ - test, ANOVA and post hock Schaffe test. The results showed that an increase in age and years of tournament play reduced the shoulder ROM of subjects $(p \leq 0.05)$. There were significant differences in shoulder ROM among athletic groups. Additionally, there are significant differences between dominant and non-dominant hands $(p \leq 0.05)$. It can be concluded that more specific stretching exercises and warm-up for shoulder in all athletes and especially in older and experienced players should be performed by coaches. The results also emphasized routine screening, corrective exercise programs and a design of preventing strategies by athletic trainers and coaches.
\end{abstract}

Keywords: Range of Motion; Shoulder Joint; Handball; Volleyball; Soccer; Age; Years of Tournament Play; Playing Post

\section{INTRODUCTION}

Joint range of motion (ROM) data is a very important reference in job and workplace design as well as in clinical diagnosis. Many factors have been reported to influence joint ROM. With an increase in age, the degeneration in these tissues becomes progressively more apparent, and results in a reduction in joint ROM. Other factors such as pain, dominant side, and the time of day may also have effects on joint ROM [1]. The physical demands of sport performance on the athletes' body cause certain musculoskeletal adaptation. Professional athletes spend most of their sporting life in training and competition [2]. Load placed on the musculoskeletal system may be generally classified as tensile or compressive. Repeated demands on a musculotendinous unit may cause it to shorten, decreasing normal joint range of motion [3]. Musculoskeletal adaptation and some special side effects due to his or her physical demands and movement patterns in professional athletes are very important subjects in sport sciences. The musculoskeletal adaptation at this point is called maladaptation, reducing joint range of motion, changing biomechanical patterns, decreasing the efficiency of force production, and increasing the chance of injuries to the musculoskeletal system [4]. Faulty posture could be also the result of that adaptation [5]. However, faulty posture does not necessarily indicate a disorder but it could cause a loss of optimum body mechanics [6].

There is a general agreement that the appropriate amount of exercise is important for the healthy growth and optimal development of the body [7]. By contrast, too much or too little exercise and inappropriate training may have some adverse effects [8]. Overtraining is an imbalance between training and recovery. This term is not new. The symptoms of prolonged fatigue, loss of motivation, burn out and staleness have been described in athletes for many decades. They are the maladaptive responses to the stimulus of training and an extended period of overload. Maladaptation flexibility due to frequent and old injuries has been recognized for some time [9]. Each sport has specific patterns of movement which has an effect on related joint range of motion (ROM) because skeletal tissue possesses an intrinsic ability to 
adapt to these physical activities. Adaptations take place in response to exercise training and those specific patterns of movements. In general, exercise programs usually include strength, power, endurance and flexibility training. In shoulder researches, measurements of range of motion (ROM) have been used as outcome measures in the vast majority of reported trials [10]. Therefore, the present study was proposed to compare the range of motion of shoulder joint (ROM) in elite athlete and non-athlete groups and its relationship to their age, post and years of tournament play.

\section{METHODOLOGY}

The subjects of this study were members of league teams with more than 2 years of play in league competitions. These subjects included 106 men with mean age (23.65 \pm 3.45 ) and mean of participation in tournament play $(5.60 \pm 2.23)$. The groups of subjects included 26 handball players (mean age $23.57 \pm 2.67$, mean playing history $6.38 \pm 2.07$ ), 25 volleyball players (mean age 22.8 \pm 4.31 , mean playing history $4.56 \pm 2.43$ ), 25 soccer players (mean age $25.08 \pm 2.53$, mean playing history $5.84 \pm 1.84$ ) and 30 non-athletes (mean age $23.23 \pm$ 3.71).

The players completed questionnaires about age, playing post, past or current shoulder injuries, duration of dominant and non-dominant glenohumeral joints were made by Leighton flexometer $(r=0.90-0.99)$ in standard position. Goniometric measurement of the glenohumeral join is difficult because of the multi-join nature of the shoulder complex. In general, the literature would appear to indicate that the Leighton flexometer is a reliable measuring tool, especially when used by a single experienced tester.

All ROMs of subjects were measured in the morning and before play or participating in warm-up drills. No goniometric measurements were taken in the afternoon or after participating in play or warm-up drills to minimize the effect of intense activity or play on range of motion. All testing took place with the subject in a standard position in special bar and plastic cast and stabilized scapula by three special wide tapes on the hip, chest and head areas. Shoulder external and internal rotations were measured with the arm positioned in $90^{\circ}$ of glenohumeral abduction and $90^{\circ}$ of elbow flexion. During testing, the subjects were asked to actively move the joint as far as possible through the range of motion (Graph 1). For each direction, three measurements were taken and the mean measurements were calculated. We performed $\mathrm{t}$ - test on dominant versus non-dominant hands of subjects for all measurements. Correlation coefficients were computed to determine the strength of the relationships among variables. We analyzed these groups
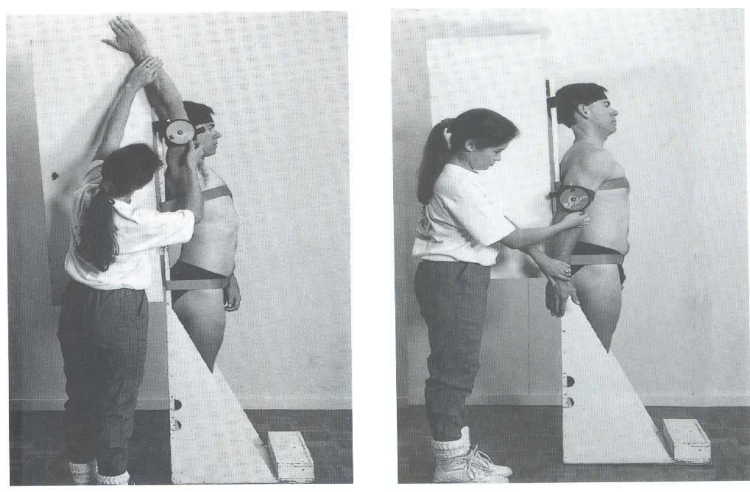

Graph 1. Flexion \& hyper extension measurements position (photo from Ackland et al. [6]).

and the relationship between shoulder ROM and age, post and history of play were analyzed by ANOVA and Post hock Scheffe test.

\subsection{Reliability of the Measuring Method}

The commonly used clinical methods for detecting muscle tightness, described by Kendall [11] are subjective. When precision and objectivity are needed, estimation of range of motion by eye (eyeballing) is too inaccurate and therefore a goniometer was used in this research. Also, standardized methods of joint motion measurement by American academy of orthopedic surgeons (AAOS) 1966 and others were advised [12]. The measuring device is always important. In addition to the accuracy of a measuring device, an instrument should be easy to be used by the tester and comfortable for the subjects. Certain joint movements, especially axial rotations are more difficult to measure than others and that with a standard universal goniometer, axial measurements are only approximates [13].

In this study all ROMs were measured with the Leighton flexometer, model WA, Spokan because it was valid, reliable, easy to use and it has been frequently used by other researchers. There are sufficient studies to prove the reliability of the Leighton flexometer: Leighton [14], Bloomfield et al. [6], and Daneshmandi [3]. The reliability and validity of this device were proved by the researchers. Ekstrand et al. [15] also used the Leighton flexometer to measure the hip ROM in soccer players.

\section{RESULTS}

The results of the present study showed that the volleyball players were also less flexible than other soccer players and non-athlete groups for internal rotation $(\mathrm{p} \leq 0.05)$.

The results of this study showed significant differences of ROM of medial rotation of the shoulder in the four 
groups of subjects included non-athletes, volleyball players, handball players and soccer players $(\mathrm{p} \leq 0.05)$ (Table 1 and Figure 1). Additionally, the results of this study showed that the ranges of motion of medial rotation of handball players were less than the other groups $(p \leq 0.05)$.

The findings of this research showed significant differences between dominant and non-dominant hands of players $(\mathrm{p} \leq 0.05)$ (Figures $2 \& 3$ ).

The amplitudes of motion of the dominant and nondominant shoulder joints in all groups were not similar and statistically there was a significant relationship between shoulder's ROMs of the dominant hand and shoulder's ROMs of the non-dominant hand. In other words, there are significant differences in external rotation between dominant and non-dominant hands of handball players $(\mathrm{p} \leq 0.05)$.

Table 1. Glenohumeral range of motions for all groups

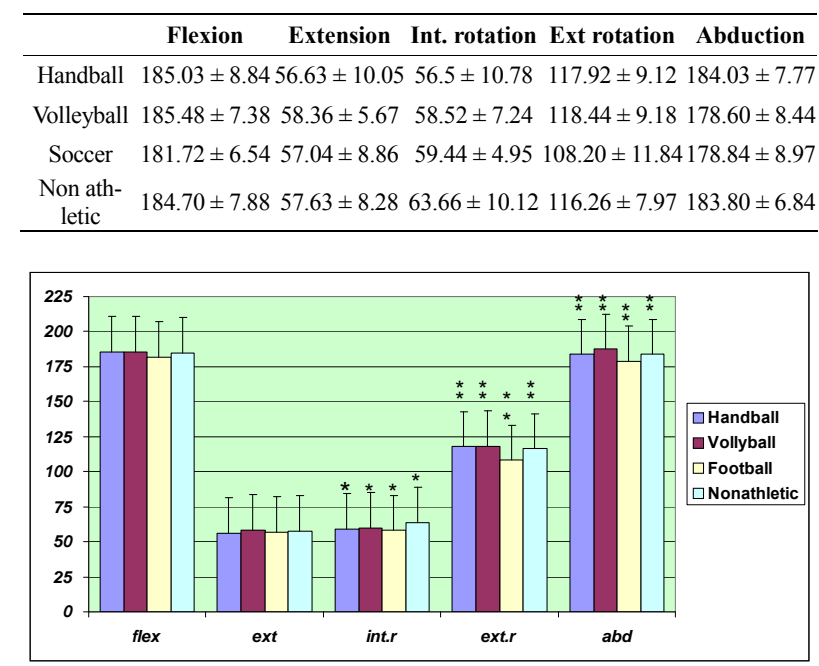

Figure 1. Glenohumeral range of movements for all groups.

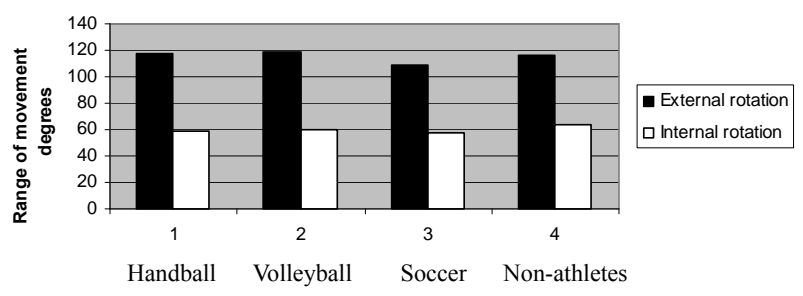

Figure 2. Glenohumeral joint rotation of dominant hand.

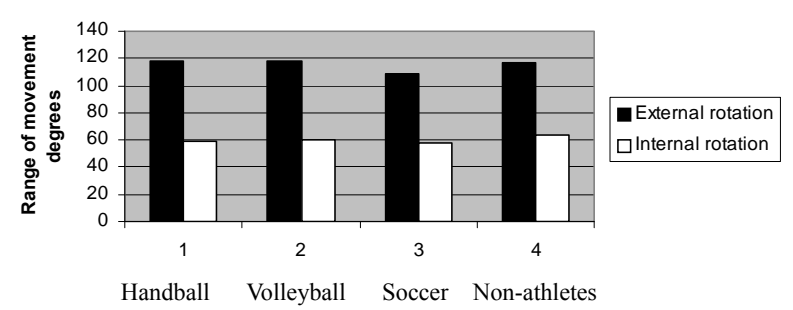

Figure 3. Glenohumeral joint rotation of non-dominant hand.
The results also showed that by increasing age, the external rotation of soccer players and non-athletes were reduced (Figure 4) and also by increasing year of tournament play, shoulder ROM decreased but this deficit not significant. There are significant differences of shoulder external and internal rotation between groups of subject and between different playing post of athletes: internal rotation in handball players $(\mathrm{p} \leq 0.05)$, internal and external rotation in volleyball players $(p \leq 0.01)$ and external rotation in soccer players $(\mathrm{p} \leq 0.05)$.

\section{DISCUSSION}

The physiologic adaptations of athletes appear to be sport specific [16]. This study was designed to measure glenohumeral range of motion, which has been implicated as a possible etiologic factor in increased genohumeral translation. Measurement of glenohumeral flexion, extension and abduction were not reported because they have not been implicated as factors in increased glenohumeral translation and because abduction also involves motion in joints other than the glenohumeral joint [17].

The results of this study showed that by increasing age and years of tournament play, internal and external rotations of shoulder in all groups were reduced. But this reduction in all groups was not significant, because range of age and years of tournament play as well as sets and time of training per week were less in the elite athletes in some countries. Increasing age and years of tournament play in athletes caused the changes in musculoskeletal structure. This adaptation may be positive or negative, for example an increase in force and masse of muscles or a change in range of motion of joints always obtained by training. Flexibility and tightness of soft tissues around joints and finally reduce range of motion

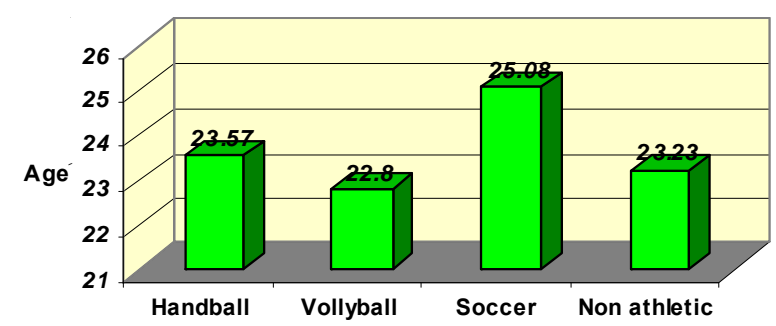

Figure 4. ROM and age.

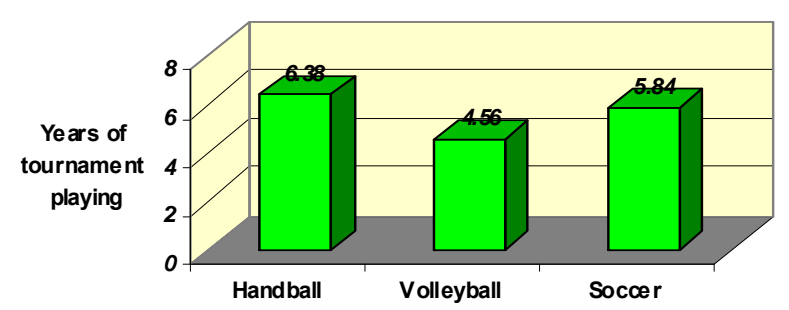

Figure 5. Mean years of history playing. 
of joints as well $[18,19]$. If the normal flexibility of the surrounding tissue of a joints is not maintained, over a period of time a decrease in joint range of motion could happen leading to a possible decrease in performance or an increase in chance of injury [4,5].

In this study, the range of external and internal shoulder rotations was different in sports and in playing posts of athletes, which can be related to their specific sport demands and emphasizes this point that flexibility is very specific in any joint, playing post and sport field. Glenohumeral internal rotation of handball and volleyball players was less than other groups in this study. A reduction in shoulder internal rotation, particularly in the dominant side, can be explained as an adaptation of the posterior shoulder musculature and capsular structure to the handball and volleyball stroke. The increase in external rotation of shoulder in these athletes is also a likely adaptation to the overhead movement in these sports. The occurrence of an excessive range of external rotation may be a product of a successful training program to increase movement and thus the range over which force can be applied to throwing or stroke $[19,20]$.

The implication of this point is that excessive external shoulder rotation can create excessive anterior humeral head displacement $[17,21,22]$. Current research has convincingly showed that deficits of internal rotation of shoulder occur as the athlete adapts to the demands of the sport. It is unclear whether these are normal adaptations that are beneficial, either locally or throughout the kinetic chain, or whether these are mal-adaptations that create potentially harmful local or kinetic chain biomechanics. Also, the role this adaptation may play in injury causation or risk is unclear. Several recent papers suggest that decreased internal rotation and total rotation may adversely affect shoulder performance, and this effect may increase the risk of injury $[22,23]$.

There are studies which show that inflexibility is a risk factor for further injury [24,25]. Achievement of full range of motion is one of the first goals in rehabilitation programs [20,22]. Most shoulder rehabilitation protocols now emphasize on a corrective exercise for internal rotation of shoulder of athletes.

The study of water polo players showed that special repetitive movement in water polo is the same as baseball and handball throwing, volleyball service and stroke and other sports with overhead movements. All these sports emphasize shoulder adduction and internal rotation that cause force, torque and mass of adductor and internal rotator muscles can lead to reduction of these ROMs [2,26].

The study of shoulder movement suggests that decreased internal and total rotation may not be optimal conditions to allow the best athletic performance with minimal risk of injury. From a performance standpoint, these conditions create inefficient biomechanics that interfere with normal coupled motion and may decrease optimal arm momentum. This paper showed degrees of asymmetry in dominant and non-dominant hands of handball and volleyball players. If we do not note it carefully and do not use correct stretch exercise program, this imbalance in musculature of shoulder girdle leads the athletes to decrease their performance or an increase in the chance of injury. For example, Young et al. [27] reported a prevalence imbalance in musculature of shoulder girdle and scoliosis in volleyball players rather than in non-athletic groups. Improvement of imbalance musculature due to hard training created scoliosis in volleyball players. Therefore, if handball and volleyball players train more and harder bilaterally, this maladaptation will be rapid.

\section{CONCLUSIONS}

Once again the results of this research showed the limitation of shoulder ROM in athletes. On the basis of this study and other investigations, it could be suggested that the specific demands, poor training, particularly lack of adequate flexibility exercises and inadequate warm-up exercises for shoulder, may be responsible for such problems. Exercising muscles without an appropriate stretching exercise program tends to decrease the motion in shoulder joints.

Very importantly, the overall (contact or non-contact sports), hard and stressful competitions, over-training, exhausting repeated movements, faulty techniques, previous injuries and probably anthropometric characteristics of players can be also considered as other factors involved in the limitation of shoulder flexibility. However, each factor will need more research.

Also, it can be recommended that more stretching exercises should be considered for older and experienced players and specific stretching exercises are recommended for different sports and playing posts. Based on the demands of any sport, coaches and athletes should apply corrective exercise programs to prevent muscular imbalance in shoulder girdle.

Much more work is needed to study the exact implications of our findings. Several directions for future study are raised by this study. Can these deficits be modified and, if so, what is the most efficient method of modification? If they are modified, what is the relationship between performance and risk of injury? Do these deficits continue to decline in a linear direction, or is there a curvilinear pattern with an absolute maximum? Were these deficits compounded by variables such as the amount of weight training exercises and previous childhood activities? 


\section{REFERENCES}

[1] Chung, M.-J. and Wang, M.-J.J. (2009) The effect of age and gender on joint range of motion of worker population in Taiwan. International Journal of Industrial Ergonomics, 39(4), 596-600.

[2] Crockett, H.C. (2002) Osseus adaptation and range of motion at the glenohumeral joint in professional baseball pitchers. American Journal of Sports Medicine, 30(1), 20-26.

[3] Hassan, D. and Shacklady, C. (2002) Hip rotation flexibility in professional athletes. International Journal of Collected Academic Articles, 1, 81.

[4] Hall, S.J. and Martin, M. (2002) Sport injury management. Williams \& Wilkins, Philadelphia.

[5] Chandler. T.J., Kibler, W.B., Uhl, T.L., Wooten, B., Kiser, A. and Stone, E. (1990) Flexibility comparisons of junior elite tennis player to other athletes. American Journal of Sports Medicine, 18(2), 134-136.

[6] Ackland, T.R., Elliott, B.C. and Bloomfield, J. (1995) Applied anatomy and biomechanics in sport. Human Kinetics Publishers, Champaign.

[7] Holland, G.J., et al. (2002) Flexibility and physical functions of older adults: A review. Journal of Aging and Physical Activity, 10(2), 169-206.

[8] Kugler, A., Krüger-Franke, M., Reininger, S., Trouillier, H.H. and Rosemeyer, B. (1996) Muscular imbalance and shoulder pain in volleyball attackers. British Journal of Sports Medicine, 30(3), 256-259.

[9] Alter, M.J. (1996) Science of stretching. Human Kinetics Publishers, Champaign.

[10] Tveitå, E.K., et al. (2008) Range of shoulder motion in patients with adhesive capsulitis; Intra-tester reproducibility is acceptable for group comparisons. BMC Musculoskeletal Disorders, 9, 49.

[11] Kendall, F., et al. (2005) Muscle testing and function with posture and pain. 5th Edition, Lippincott Williams \& Wilkins Publisher, Philadelphia.

[12] Norkin, C.C. and White, D.J. (1995) Measurement of joint motion: A guide to goniometry. 2nd Edition, F. A. Davis Company, Philadelphia.

[13] Ellenbecker, T.S., et al. (1996) Glenohumeral joint internal and external rotation range of motion in elite junior tennis players. Journal of Orthopaedic \& Sports Physical Therapy, 24(6), 336-341.
[14] Leighton, J.R. (1957) Flexibility characteristics of three specialized skill groups of champion athletes. Archives of Physical Medicine and Rehabilitation, 38(9), 580-583.

[15] Ekstrand, J., et al. (1982) Lower extremity goniometric measurement: A study to determine their reliability. Archives of Physical Medicine and Rehabilitation, 63(4), 171-175.

[16] Harryman, D.T., et al. (1992) The role of the rotator interval capsule in passive motion and stability of the shoulder. The Journal of Bone and Joint Surgery, 74(1), 53-66.

[17] Reagan, K.M., et al. (2002) Humeral retroversion and its relationship to glenohumeral rotation in the shoulder of college baseball player. American Journal of Sports Medicine, 30(3), 354-360.

[18] Leatt, P., et al. (1987) Specific muscular development in under-18 soccer player. Journal of Sports Sciences, 5(2), $165-175$.

[19] Herington, L., et al. (1998) Glenohumeral joint: Internal and external rotation range of motion in javelin throwers. British Journal of Sports Medicine, 32(3), 226-228.

[20] Silliman, J.F. and Hawkins, R.J. (1991) Current concepts and recent advances in the athlete's shoulder. Clinics in Sports Medicine, 10(4), 693-705.

[21] Wilk, K.E., et al. (2002) Current concepts in the rehabilitation of the overhead throwing athlete. American Journal of Sports Medicine, 30(1), 136-151.

[22] Wilk, K.E., et al. (1997) Current concept: The stabilizing structures of the glenohumeral joint. Journal of Orthopaedic \& Sports Physical Therapy, 25(6), 364-379.

[23] Kibler, W.B. (1995) Clinical examination of the shoulder: Athletic injuries of the shoulder. McGraw-Hill, Inc., New York.

[24] Kibler, W.B., Chandler, T.J. and Pace, B.K. (1992) Principles of rehabilitation after chronic tendon injuries. Clinics in Sports Medicine, 11(3), 661-671.

[25] Pieper, H.G. (1998) Humeral torsion in the throwing arm of handball players. American Journal of Sports Medicine, 26(2), 247-253.

[26] McMaster, W.C., et al. (1992) Shoulder torque changes in the swimming athlete. American Journal of Sports Medicine, 20(3), 323-327.

[27] Kim, Y.K., et al. (2003) The relationship between humeral retroversion and shoulder rotation in maggot league baseball pitchers. 5th Asian Federation of Sports Medicine Congress, Seoul, 15 September 2003. 